\title{
Photons from strange-quark annihilation in a quark-gluon plasma
}

\author{
G. Staadt and W. Greiner \\ Institut für Theoretische Physik der J. W. Goethe-Universität, D-6000 Frankfurt am Main 11, \\ Postfach 111932, West Germany
}

\author{
J. Rafelski \\ Institute of Theoretical Physics and Astrophysics, University of Cape Town, Rondebosch 7700, Republic of South Africa
}

(Received 20 February 1985)

\begin{abstract}
We compute the energy spectrum of photons which originate from the quark-annihilation process $s \bar{s} \rightarrow \gamma g$ in quark-gluon plasma. The spectrum peaks at an energy $E_{\gamma}^{\max } \sim 2 m_{s} \sim 400 \mathrm{MeV}$ in the rest frame of the plasma. We expect one photon from the above process in the energy range of $2 m_{s} \pm 0.25 m_{s}$ per hundred quark-gluon plasmas of a size $R=3 \mathrm{fm}$ and a lifetime $\tau=6 \mathrm{fm} / c$ formed in nuclear collisions.
\end{abstract}

\section{INTRODUCTION}

Given the present knowledge about strong interaction, one has to assume that in collisions of heavy nuclei with sufficiently high kinetic energy, a high-energy-density region can be formed in which the hadrons dissolve into their constituents, i.e., into quark-gluon plasma. ${ }^{1}$ Several estimates $^{2}$ lead to $0.5-2 \mathrm{GeV} / \mathrm{fm}^{3}$ for the critical energy density. The quark-gluon plasma represents a new physical phase and its physics can often approximately be described by methods of perturbation theory. ${ }^{1,2}$ In order to verify this hypothesis of the existence of a new phase of matter one has to consider all processes, which could give a hint to the existence of the plasma. In this paper we consider certain processes leading to high-energy photons. These photons will, in general, leave the interaction region without further interactions.

Numerous photon production processes which have little to do with the plasma state will normally dominate the photon spectra. However, one can perform an in-depth study of various contributions ${ }^{3}$ and it is conceivable that even a small effect may become visible in certain kinematic domains, such as small $x$, large $P_{1}$, once a correlation with strangeness overabundance is established.

In the plasma phase, numerous pairs of strange and antistrange quarks are created by gluons, ${ }^{4}$ which occasionally annihilate again. One possible, though rare, annihilation process is to create a gluon and a photon. Naively one expects that these photons possess a relatively high energy. The energy of photons is given by $E_{\gamma}=E_{s}+E_{\bar{s}}-E_{g}$ and it should be recorded that strange quarks and antiquarks carry their rest mass, in addition to the thermal kinetic energy, into the reaction while emission of low-energy gluons is stimulated by the existence of numerous low-energy gluons in plasma. In this paper we calculate the spectrum of the photons which are produced in such processes $(s \bar{s} \rightarrow \gamma g)$ quantitatively to first order in the perturbative approach.

\section{TRANSITION PROBABILITY: $s \bar{s} \rightarrow \gamma g$}

First we compute the transition probability per unit time and volume $(\omega)$ of the process $s \bar{s} \rightarrow \gamma g$. For that purpose we use the lowest-order perturbative QCD and QED. This means that we evaluate the Feynman diagrams shown in Fig. 1.

The symbols indicated in this figure have the following meaning: $p_{s}, p_{\bar{s}}, q, k$ are the four-momenta of the particles, $s_{1}, s_{2}$ the spin of the quarks, $i, j$ the quark color, $a$ the gluon color, $\epsilon^{P}, \epsilon^{G}$ the polarization vectors.

Using the nomenclature of Ref. 5 the transition probability $\omega$ per unit time and volume can be written in terms of the invariant amplitude $\mathscr{M}$ as

$\omega=(2 \pi)^{4} \delta^{4}\left(p_{s}+p_{\bar{s}}-q-k\right) \frac{1}{V^{4}} \frac{m^{2}}{4 E_{s} E_{\bar{s}} E_{g} E_{\gamma}}|\mathscr{M}|^{2}$.

The two contributions shown in Fig. 1

$$
\mathscr{M}=\mathscr{M}^{1}+\mathscr{M}^{2}
$$

are given explicitly by ${ }^{6}$

$$
\begin{aligned}
\mathscr{M}^{1}= & \bar{v}^{j}\left(p_{\bar{s}}, s_{2}\right)(-i g) \epsilon^{G} T_{j i}^{a} \frac{i}{p_{s}-q-m+i \epsilon} \\
& \times\left(-\frac{1}{3} i e\right) \epsilon^{p} u^{i}\left(p_{s}, s_{1}\right), \\
\mathscr{M}^{2}= & \bar{v}^{j}\left(p_{\bar{s}}, s_{2}\right)\left(-\frac{1}{3} i e\right) \epsilon^{p} \frac{i}{p_{s}-\not k-m+i \epsilon} \\
& \times(-i g) \epsilon^{G} T_{j i}^{a} u^{i}\left(p_{s}, s_{1}\right) .
\end{aligned}
$$

We find that the propagator of the virtual quark is not dependent on the presence of the other plasma particles, because the chemical potential of the strange quarks is zero. The chemical potential must be zero, since strange quarks are always produced in pairs. ${ }^{2}$ This independence can be established as follows: A fermion propagator in momentum space can be written ${ }^{7}$ 


$$
\begin{aligned}
S_{F}(p)= & \frac{p^{\prime}+m}{p^{\prime 2}-m^{2}+i \epsilon} \\
& +\frac{\not p^{\prime}+m}{e^{(E-\mu) / T}+1} 2 \pi i \delta\left(p^{\prime 2}-m^{2}\right),
\end{aligned}
$$

with

$$
p^{\prime \alpha}=p^{\alpha}+\mu u^{\alpha}
$$

( $\mu=$ chemical potential). For strange quarks we have $\mu_{s}=0$ and Eq. (5) leads to $p^{\prime \alpha}=p^{\alpha}$. From kinematic considerations we know that $p^{2}$ must always be unequal to zero in order to have a massless photon emitted. Therefore the second term in Eq. (4) does not yield a contribution and Eq. (4) reduces to the propagator used in Eqs. (3). Let us here further note that these considerations are not valid for the light ( $u$ and $d$ ) quarks, since their chemical potentials do not vanish in general.

We evaluate $|\mathscr{M}|^{2}$ in the rest frame of the $s$ quark using the radiation gauge. The result is

$$
\begin{aligned}
\sum|\mathscr{M}|^{2}=\frac{8}{9} \frac{e^{2} g^{2}}{m^{2}} & \mid \frac{|\mathbf{k}|}{|\mathbf{q}|}+\frac{|\mathbf{q}|}{|\mathbf{k}|} \\
& +\frac{2 m(|\mathbf{q}|+|\mathbf{k}|)}{|\mathbf{q}||\mathbf{k}|}-\frac{m^{2}}{|\mathbf{k}|^{2}} \\
& \left.-\frac{m^{2}}{|\mathbf{q}|^{2}}-\frac{2 m^{2}}{|\mathbf{q}||\mathbf{k}|}\right) .
\end{aligned}
$$

$\sum$ means the sum over the spin/color quantum numbers of all particles. Expression (6) is valid only in the rest frame of the $s$ quark. However, we can transform Eq. (6) into a function of two invariant Mandelstam variables. Then the resulting expression is valid in every Lorentz system. The three Mandelstam variables of the process

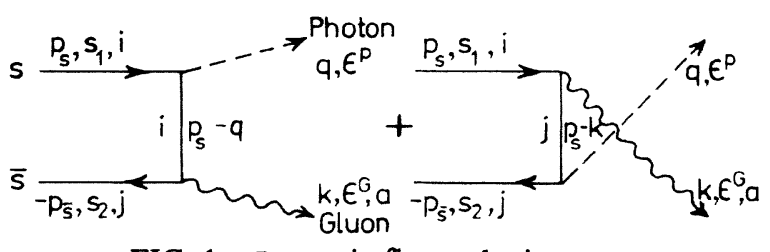

FIG. 1. $s \bar{s} \rightarrow \gamma g$ in first order in $\alpha_{s} \alpha$.

described by Fig. 1 are

$$
\begin{aligned}
& s=\left(p_{s}+p_{\bar{s}}\right)^{2}, \\
& t=\left(p_{s}-k\right)^{2}, \\
& u=\left(p_{s}-q\right)^{2},
\end{aligned}
$$

and are constrained by

$$
s+t+u=2 m^{2} \text {. }
$$

We make use of $s$ and $t$ as independent variables and obtain the final formula:

$$
\begin{aligned}
\sum|\mathscr{M}|^{2}=\frac{8}{9} \frac{e^{2} g^{2}}{m^{2}} & \frac{\left(m^{2}-t\right)}{\left(s+t-m^{2}\right)}+\frac{\left(s+t-m^{2}\right)}{\left(m^{2}-t\right)} \\
& -\frac{4 m^{2}\left(-s+2 m^{2}\right)}{\left(m^{2}-t\right)\left(s+t-m^{2}\right)}-\frac{4 m^{4}}{\left(m^{2}-t\right)^{2}} \\
& \left.-\frac{4 m^{4}}{\left(s+t-m^{2}\right)^{2}}\right] .
\end{aligned}
$$

In Eq. (8) the QCD coupling constant $\alpha_{s}=g^{2} / 4 \pi$ is also a slowly changing function of $s$ and $t$. However, we will use a constant value of $\alpha_{s}$ in further calculations which is to be seen as a weighted average over space- and timelike transferred momenta.

\section{PHOTON SPECTRUM}

The number of events $(s \bar{s} \rightarrow \gamma g)$ per unit time and volume is given by ${ }^{8}$

$$
A=\frac{d N}{d^{3} x d t}=\int d^{3} p_{s} d^{3} p_{\bar{s}} d^{3} k d^{3} q \frac{m^{2}}{(2 \pi)^{8} 4 E_{s} E_{\bar{s}} E_{g} E_{\gamma}} \rho\left(p_{s}\right) \rho\left(p_{\bar{s}}\right)\left[1+\rho_{B}(k)\right] \delta^{4}\left(p_{s}+p_{\bar{s}}-k-q\right) \sum|\mathscr{M}|^{2} \text {. }
$$

$\rho\left(p_{s}\right), \rho\left(p_{s}\right)$, and $\rho_{B}(k)$ are the distribution functions of the corresponding particles. In order to obtain these functions, we make the simplest possible assumption, i.e., that quarks and gluons in the plasma can be described as an (nearly) ideal gas. ${ }^{1,2}$ Thus for the emitted gluons we include the Bose function $\rho_{B}(k)$ and notice the appearance of the stimulated process $\left(\sim \rho_{B}\right)$ next to the spontaneous one $(\sim 1)$ in the square brackets. The present consideration of the quantum statistics effect-the induced reaction $\left(\sim \rho_{B}\right)$-goes beyond the benign neglect of these types of corrections, such as the neglect of Pauli blocking of strange-quark formation in Ref. 4. We shall see that even the very much greater gluon density occurring here will not significantly affect the computed reaction rate.

The quark densities $\rho\left(p_{s}\right)$ and $\rho\left(p_{\bar{s}}\right)$ follow the Fermi distribution functions; in the rest frame of the plasma we obtain

$$
\left(e^{E / T}+1\right)^{-1}=e^{-E / T}-e^{-2 E / T}+\cdots, E>0 .
$$

For both $\bar{s}$ and $s$ we use only the first term of the series, which corresponds to the classical Boltzmann distribution. The overestimation made by this approach is greatest at the lowest possible energy (the mass) of the $s$ and $\bar{s}$ quark, where we have for both quarks $E / T \approx 1$. Since Boltzmann approximation simplifies the calculation greatly we have used it also in view of the other uncertainties prevailing here.

We are interested in the photon spectrum and we will first compute the Lorentz-invariant quantity $E_{\gamma} d^{3} A / d^{3} q$. Since $\sum|\mathscr{M}|^{2}$ is a function of $s$ and $t$, we introduce integrations over these variables: 


$$
\begin{aligned}
E_{\gamma} \frac{d^{3} A}{d^{3} q}=\int d^{3} p_{s} \int d^{3} p_{\bar{s}} \int & d^{3} k \frac{m^{2}}{(2 \pi)^{8} 4 E_{s} E_{\bar{s}} E_{g}} \rho\left(p_{s}\right) \rho\left(p_{\bar{s}}\right)\left[1+\rho_{B}(k)\right] \\
& \times \delta^{4}\left(p_{s}+p_{\bar{s}}-q-k\right) \int d s \delta\left[s-\left(p_{s}+p_{\bar{s}}\right)^{2}\right] \int d t \delta\left[t-\left(p_{s}-k\right)^{2}\right] \sum|\mathscr{M}|^{2}(s, t)
\end{aligned}
$$

We now perform the following operations.

(1) $\int d^{3} k\left(2 E_{g}\right)^{-1} \rightarrow \int d^{4} k \delta\left(k^{2}\right) \theta\left(k_{0}\right)$

(2) $\int d^{4} k$ is carried out using the $\delta^{4}$ function and we set $k=p_{s}+p_{\bar{s}}-q$.

(3) We intend to carry out first the integrals over $p_{s}$ and $p_{\bar{s}}$. Then we obtain, where $t_{ \pm}=m^{2}-\frac{1}{2} s \pm\left(s^{2} / 4-m^{2} s\right)^{1 / 2}$

$$
\begin{aligned}
& E_{\gamma} \frac{d^{3} A}{d^{3} q}=\frac{m^{2}}{2(2 \pi)^{8}} \int_{4 m^{2}}^{\infty} d s \int_{t_{-}}^{t^{+}} d t \sum|\mathscr{M}|^{2}(s, t) \int \frac{d^{3} p_{s}}{E_{s}} \int \frac{d^{3} p_{\bar{s}}}{E_{\bar{s}}} \rho\left(p_{s}\right) \rho\left(p_{\bar{s}}\right)\left[1+\rho_{B}\left(p_{s}+p_{\bar{s}}-q\right)\right] \\
& \times \delta\left(s+t-m^{2}-2 p_{s} \cdot q\right) \theta\left(E_{s}+E_{\bar{s}}-E_{\gamma}\right) \\
& \times \delta\left(t-m^{2}+2 p_{\bar{s}} \cdot q\right) \delta\left(s-2 m^{2}-2 p_{s} \cdot p_{\bar{s}}\right) .
\end{aligned}
$$

We evaluate $p_{s}, p_{\bar{s}}$ integrals in the rest frame of the plasma where the statistical distribution functions take a simple form. We notice that (1) $d^{3} p \rightarrow|\mathbf{p}| E d E d \Omega$ for $p_{s}$ and $p_{\bar{s}}$, (2) three angular integrals are canceled by the $\delta$ functions and the last angular integral yields a factor $4 \pi$, and (3) the cancellation of the three angular integrals by the $\delta$ functions leads to restrictions of the domains of integration for $E_{s}$ and $E_{\bar{s}}$. The result of these operations is given by the following expression:

$$
\begin{gathered}
E_{\gamma} \frac{d^{3} A}{d^{3} q}=\frac{m^{2} \pi}{4(2 \pi)^{8} E_{\gamma}} \int_{4 m^{2}}^{\infty} d s \int_{t_{-}}^{t} d t \sum|\mathscr{M}|^{2}(s, t) \int_{B} d E_{s} \int_{B^{\prime}} d E_{\bar{s}} e^{-\left(E_{s}+E_{\bar{s}}\right) / T}\left(1+\frac{1}{e^{\left(E_{s}+E_{\bar{s}}-E_{\gamma}\right) / T}-1}\right) \\
\times \frac{1}{\left(a E_{\bar{s}}{ }^{2}+b E_{\bar{s}}+c\right)^{1 / 2}},
\end{gathered}
$$

where the integration regions $\{B\},\left\{B^{\prime}\right\}$ and constants $a, b, c$ are defined in Appendix A [see Eqs. (A9), (A10), and (A5)-(A7)]. Using the substitution $x=E_{s}+E_{\bar{s}}, y=E_{\bar{s}}$ the two integrals over $E_{s}$ and $E_{\bar{s}}$ can be reduced to

$$
\int_{B} d E_{s} \int_{B^{\prime}} d E_{\bar{s}} \cdots=\frac{2 \pi}{s} \int_{s / 4 E_{\gamma}+E_{\gamma}}^{\infty} d x e^{-x / T}\left(1+\frac{1}{e^{\left(x-E_{\gamma}\right) / T}-1}\right) .
$$

The spontaneous emission (SE) [corresponds to 1 in the large parentheses in Eq. (14)] can now be obtained analytically. The integrations over $x$ and $t$ [Eqs. (13) and (14)] are carried out analytically and the remaining function of $s$ can be approximated by a simplified function of which analytical integrals exist. We obtain the simplified function from the investigation of the asymptotic behavior of the exact function, where we can replace, e.g.,

$$
\sqrt{1-4 / s} \underset{s \rightarrow \infty}{\sim} 1, \ln \left(\frac{1+\sqrt{1-4 / s}}{1-\sqrt{1-4 / s}}\right) \underset{s \rightarrow \infty}{\sim} \ln s
$$

As it turns out we can use these approximations in the total domain of integration. The error of the final result introduced in this way is of the order of magnitude of $1 \%$ and thus negligible. The result for the spontaneous emission is

$$
\begin{aligned}
\left\{E_{\gamma} \frac{d^{3} A}{d^{3} q}\right]_{\mathrm{SE}}=3.313 \times 10^{-7} g^{2} m^{2} \frac{T}{E_{\gamma}} e^{-E_{\gamma} / T}\{ & \left(-2.772+1.545 \frac{E_{\gamma} T}{m^{2}}\right) e^{-m^{2} / E_{\gamma} T} \\
& -\left[3.545+4 \frac{E_{\gamma} T}{m^{2}}+2.772 \frac{m^{2}}{E_{\gamma} T}\right] \mathrm{Ei}\left[-\frac{m^{2}}{E_{\gamma} T}\right) \\
& \left.+\left(2-\frac{m^{2}}{E_{\gamma} T}\right) e^{m^{2} / E_{\gamma} T}\left[\mathrm{Ei}\left(-\frac{m^{2}}{E_{\gamma} T}\right)\right]^{2}+2 e^{m^{2} / E_{\gamma} T} \mathrm{Ei}\left[-\frac{2 m^{2}}{E_{\gamma} T}\right)\right\} \\
=f_{1}\left(g^{2}, m^{2}, T, E_{\gamma}\right) . &
\end{aligned}
$$


We now turn to the calculation of the reduced emission (IE) [corresponds to the Bose function in the large parentheses in Eq. (14)]. For small values of $\left(x-E_{\gamma}\right) / T$ the Bose function can be expanded in a power series in $\left(x-E_{\gamma}\right) / T$ :

$$
\begin{aligned}
\frac{1}{e^{\left(x-E_{\gamma}\right) / T}-1}= & {\left[\frac{x-E_{\gamma}}{T}\right]^{-1}-\frac{1}{2}+\frac{B_{1}}{2 !} \frac{\left(x-E_{\gamma}\right)}{T} } \\
& -\frac{B_{2}}{4 !}\left[\frac{x-E_{\gamma}}{T}\right]^{3}+\cdots
\end{aligned}
$$

where $B_{i}$ are the Bernoulli numbers. For large values of $\left(x-E_{\gamma}\right) / T$ (i.e., large gluon energies) the induced radiation has a very small yield and can be neglected. We thus can replace the Bose function by the approximate expression

$$
\begin{aligned}
(16) \approx & {\left[\left[\frac{x-E_{\gamma}}{T}\right]^{-1}-\frac{1}{2}+\frac{1}{12} \frac{x-E_{\gamma}}{T}\right] } \\
& \times \theta\left(3.3 T+E_{\gamma}-x\right) .
\end{aligned}
$$

We have verified that for all values of $E_{\gamma}$ the error of the final result made by this approximation is only of the order of magnitude of $1 \%$. We now can perform the integrations over $x$ and $t$ analytically. We obtain

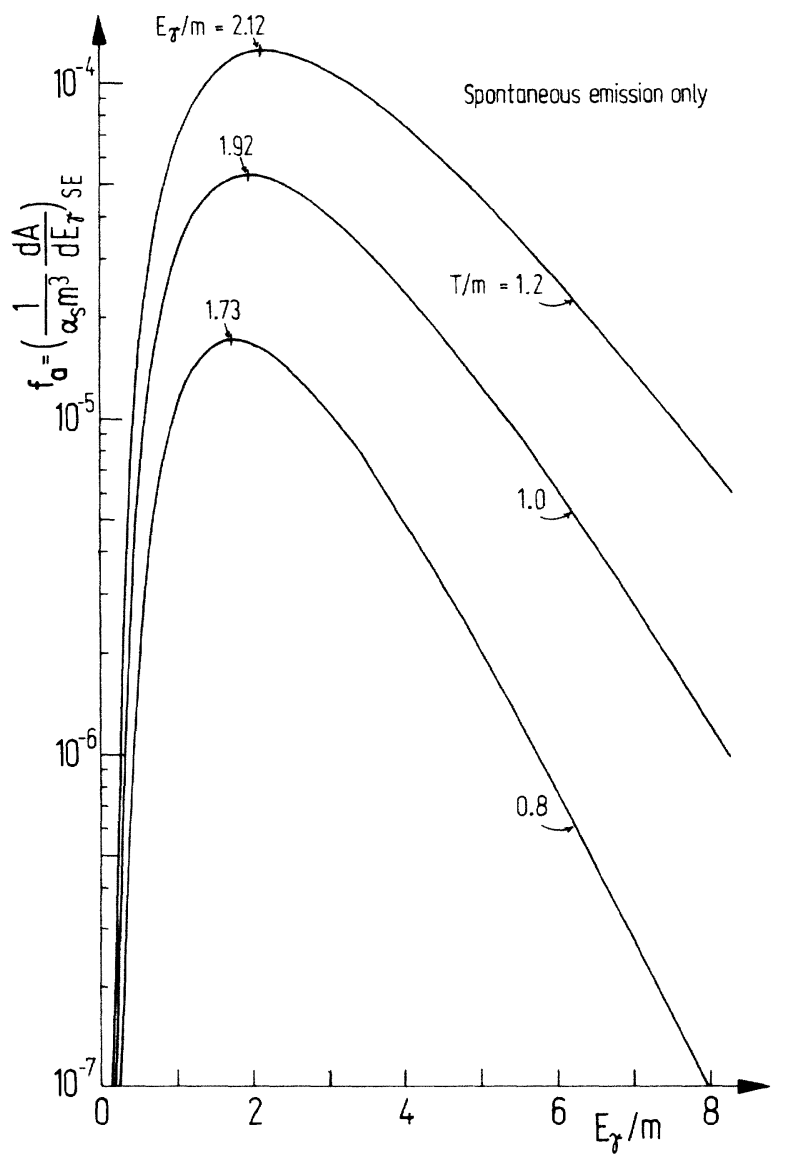

FIG. 2. Energy spectrum (spontaneous emission) in the rest frame of the plasma. $m$ is the (strange-) quark mass.

$$
\begin{aligned}
{\left[E_{\gamma} \frac{d^{3} A}{d^{3} q}\right]_{\mathrm{IE}}=} & \frac{e^{2} g^{2}}{9(2 \pi)^{6}} \frac{T}{E_{\gamma}} e^{-E_{\gamma} / T} \theta\left(E_{\gamma}-\frac{m^{2}}{3.3 T}\right) \\
& \times \int_{4 m^{2}}^{13.2 E_{\gamma} T} d s \frac{1}{s} v(s) w(s),
\end{aligned}
$$

with

$$
\begin{aligned}
& v(s)=\mathrm{Ei}(-3.3)+\frac{1.7}{12} e^{-3.3}-\mathrm{Ei}\left(-\frac{s}{4 E_{\gamma} T}\right) \\
& -\frac{s}{12} e^{-s /\left(4 E_{\gamma} T\right)}+\frac{1}{12} e^{-s /\left(4 E_{\gamma} T\right)} \frac{s}{4 E_{\gamma} T}, \\
& w(s)=-2 s\left(1-\frac{4 m^{2}}{s}\right)^{1 / 2}-8 m^{2}\left(1-\frac{4 m^{2}}{s}\right)^{1 / 2} \\
& +2 s \ln \left(\frac{1+\left(1-\frac{4 m^{2}}{s}\right)^{1 / 2}}{1-\left(1-\frac{4 m^{2}}{s}\right)^{1 / 2}}\right) \\
& +8 m^{2} \ln \left(\frac{1+\left(1-\frac{4 m^{2}}{s}\right)^{1 / 2}}{1-\left(1-\frac{4 m^{2}}{s}\right)^{1 / 2}}\right) \\
& -\frac{16 m^{4}}{s} \ln \left(\frac{1+\left(1-\frac{4 m^{2}}{s}\right)^{1 / 2}}{1-\left(1-\frac{4 m^{2}}{s}\right)^{1 / 2}}\right)
\end{aligned}
$$

However, we have not found a useful approximant for the remaining $s$ integral which must be evaluated numerically. The result assumes the form

$$
\left(E_{\gamma} \frac{d^{3} A}{d^{3} q}\right]_{\mathrm{IE}}=f_{2}\left(g^{2}, m^{2}, T, E_{\gamma}\right) .
$$

$E_{\gamma} d^{3} A / d^{3} q$ is a Lorentz-invariant quantity, which takes the form of Eq. (15) plus Eq. (21) in the rest frame of the plasma. In this reference system the spectrum $d^{3} A / d^{3} q$ depends only on $|\mathrm{q}|=E_{\gamma}$, i.e., $d^{3} q=d \Omega E_{\gamma}{ }^{2} d E_{\gamma}$. Therefore we obtain the energy spectrum in the rest frame of the plasma as

$$
\frac{d A}{d E_{\gamma}}=4 \pi E_{\gamma}\left(f_{1}+f_{2}\right) \text {. }
$$

Substitution of $g^{2}=4 \pi \alpha_{s}$ and

$$
f_{1}=\left(\alpha_{s} m^{3} / 4 \pi E_{\gamma}\right) f_{a}\left(T / m, E_{\gamma} / m\right)
$$

and

$$
f_{2}=\left(\alpha_{s} m^{3} / 4 \pi E_{\gamma}\right) f_{b}\left(T / m, E_{\gamma} / m\right)
$$

into Eq. (22) yields

$$
\frac{1}{\alpha_{s} m^{3}} \frac{d A}{d E_{\gamma}}=f_{a}+f_{b} \text {. }
$$




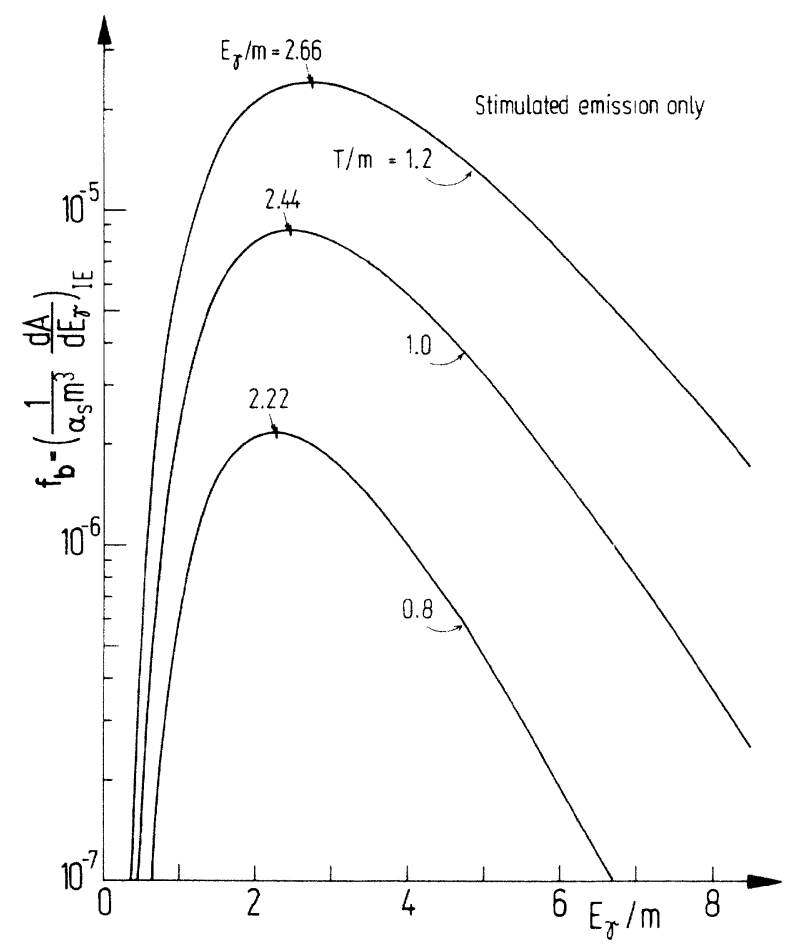

FIG. 3. Energy spectrum (induced emission) in the rest frame of the plasma. $m$ is the (strange-) quark mass.

In Figs. 2, 3, and 4 we show $f_{a}, f_{b}$ and $f_{a}+f_{b}$ as functions of $E_{\gamma} / \mathrm{m}$ for different values of $T / \mathrm{m}$.

\section{DISCUSSION OF RESULTS}

The position of photon peaks from induced and spontaneous processes shown in Figs. 2 and 3 confirms the qualitative argument made in the Introduction: the induced peaks are shifted to higher energies by roughly 0.5 $\mathrm{m}$ in the range of temperatures considered here $(0.8<\mathrm{T}<1.2 \mathrm{~m})$. However, the induced photons turn out to be about four times less numerous and hence the photon spectrum is dominated by the spontaneous process as shown in Fig. 4.

Considering the slopes of the spectra we find that in general they obey an $e^{-E /(\kappa T)}$ law, where $\kappa$ ranges from 1.15 to 1.3 . Hence a naive approach in which slopes are used as a measure of the temperature would result in an overestimation by a factor $\kappa$ of the prevailing plasma temperature. Furthermore, the $\kappa$ 's of the induced spectra are about 5\% larger than those of the spontaneous radiation.

We recall now that $A$ is the rate per unit of volume and time. To find out the number of photons per unit of energy coming from a quark-gluon plasma, we have to multiply Eq. (23) with the volume $V$ of the plasma and its estimated lifetime $\tau$ :

$$
\left.\frac{d n_{\gamma}}{d E_{\gamma}}\right|_{\text {event }}=V \tau m^{3} \alpha_{s}\left(f_{a}+f_{b}\right) .
$$

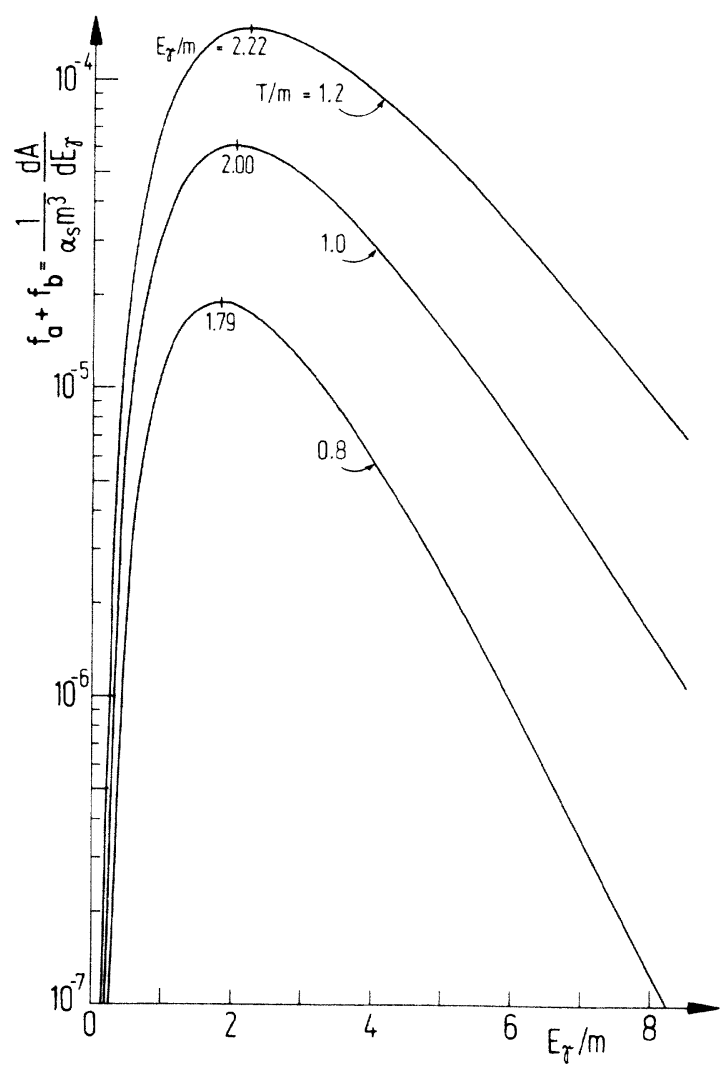

FIG. 4. Energy spectrum (spontaneous + induced emission) in the rest frame of the plasma. $m$ is the (strange-) quark mass.

For $V \approx 4 \pi / 3(3 \mathrm{fm})^{3}$ and $\tau=6 \mathrm{fm} / c$ and assuming $m=200 \mathrm{MeV}, \alpha_{s}=\frac{1}{2}$ the normalized spectra (Figs. 2-4) have to be multiplied with a factor $1.8(\mathrm{MeV})^{-1}$ in order to obtain the proper values. We see that for $T=200 \mathrm{MeV}$ in 100 quark-gluon plasma events one photon from $s \bar{s}$ annihilation will emerge in an energy range of $\Delta E_{\gamma}=100$ $\mathrm{MeV}$ around $E_{\gamma}^{\max }=2 m_{s}$.

Integrating our spectra we find one photon from $s \bar{s}$ annihilation per ten plasma events living $6 \mathrm{fm} / c$ and having a radius of $3 \mathrm{fm}$. Thus we conclude that high-energy photons from $s \bar{s}$ annihilation in quark-gluon plasma are rare.

Finally, one may use our results, with all the due reservations, also as an order-of-magnitude estimate for the rate of high-energy photon radiation from light $u, d$ quarks. Setting $T=200 \mathrm{MeV}, m \approx 10-20 \mathrm{MeV}$, which is appropriate to light quarks, we find that the number of emitted photons is enhanced roughly by a factor 10 . The peaks are located at an energy of $E_{\gamma}^{\max } \approx 235-244 \mathrm{MeV}$. However, one should remember that light-quark processes are modified due to modifications of the quark propagator and quark distribution functions at finite baryon density. Furthermore, the up quarks have a four times larger coupling to the photon field.

Of course Compton-type processes must also be considered for photon production in quark-gluon plasma. However, our expectation has been that high-energy photons are dominated by annihilation diagrams studied in this paper. 


\section{APPENDIX A}

Three angular integrals in Eq. (12)

$$
\left(d^{3} p_{s, \bar{s}} \rightarrow\left|\mathbf{p}_{s, \bar{s}}\right| E d E d \Omega\right)
$$

are carried out using the $\delta$ functions. These operations lead to the following restrictions of the domains of integration for $E_{s}$ and $E_{\bar{s}}$ :

$$
\begin{aligned}
& E_{s} \geq \frac{\left(s+t-m^{2}\right)}{4 E_{\gamma}}+\frac{m^{2} E_{\gamma}}{\left(s+t-m^{2}\right)}, \\
& E_{\bar{s}} \geq \frac{\left(m^{2}-t\right)}{4 E_{\gamma}}+\frac{m^{2} E_{\gamma}}{\left(m^{2}-t\right)}, \\
& E_{\bar{s}} \leq-\frac{b}{2 a}+\left[\frac{b^{2}-4 a c}{4 a^{2}}\right)^{1 / 2}, \\
& E_{\bar{s}} \geq-\frac{b}{2 a}-\left[\frac{b^{2}-4 a c}{4 a^{2}}\right]^{1 / 2},
\end{aligned}
$$

where

$$
\begin{aligned}
& a=-\frac{\left(s+t-m^{2}\right)^{2}}{4}, \\
& b=E_{s} \frac{\left(s+t-m^{2}\right)\left(m^{2}-t\right)}{2}+E_{\gamma} \frac{s}{2}\left(s+t-3 m^{2}\right),
\end{aligned}
$$

$$
\begin{aligned}
c= & E_{\gamma}{ }^{2}\left(-\frac{1}{4} s^{2}+m^{2} s\right)+\frac{s}{4}\left(s t+t^{2}+m^{4}-2 t m^{2}\right) \\
& -\frac{\left(m^{2}-t\right)^{2}}{4} E_{s}{ }^{2}-E_{s} E_{\gamma} \frac{s}{2}\left(m^{2}+t\right) .
\end{aligned}
$$

Equation (12) further satisfies the kinematic constraint

$$
E_{\bar{s}} \geq E_{\gamma}-E_{s} \text {. }
$$

A study of the conditions Eqs. (A1)-(A4) and (A8) shows that it is sufficient to require Eqs. (A1), (A3), and (A4). Then Eqs. (A2) and (A8) are always fulfilled. We intend to carry out first the $E_{\bar{s}}$ integration and after that the integration over $E_{s}$. Hence we obtain the following domains of integration:

$$
\begin{aligned}
& \int d E_{\bar{s}}: \text { from }-\frac{b}{2 a}-\left(\frac{b^{2}-4 a c}{4 a^{2}}\right)^{1 / 2} \text { to }-\frac{b}{2 a}+\left(\frac{b^{2}-4 a c}{4 a^{2}}\right)^{1 / 2}=\left\{B^{\prime}\right\}, \\
& \int d E_{s}: \text { from } \frac{\left(s+t-m^{2}\right)}{4 E_{\gamma}}+\frac{m^{2} E_{\gamma}}{\left(s+t-m^{2}\right)} \text { to } \infty=\{B\} .
\end{aligned}
$$

1S. A. Chin, Phys. Lett. 78B, 552 (1978); J. Rafelski and R. Hagedorn, in Thermodynamics of Quarks and Hadrons, edited by H. Satz (North-Holland, Amsterdam, 1981); G. Domokos and J. I. Goldman, Phys. Rev. D 23, 203 (1981).

2See, e.g., J. Rafelski and M. Danos, in Lecture Notes in Physics 231, edited by W. D. Heiss (Springer, New York, 1985), p. 361; B. Müller, Universität Frankfurt Report No. UFTP 125/83 (unpublished), and references therein.
${ }^{3}$ P. V. Chliapnikov et al., Phys. Lett. 141B, 276 (1984).

4J. Rafelski and B. Müller, Phys. Rev. Lett. 48, 1066 (1982).

5J. D. Bjorken and S. D. Drell, Relativistic Quantum Mechanics (McGraw-Hill, New York, 1964).

${ }^{6}$ R. Cutler and D. Sivers, Phys. Rev. D 17, 196 (1978) and Ref. 5.

${ }^{7}$ See, e.g., H. A. Weldon, Phys. Rev. D 26, 1394 (1982).

${ }^{8}$ See, e.g., Ref. 5. 